\title{
Four-color fluorescence in-situ hybridization is useful to assist to distinguish early stage acral and cutaneous melanomas from dysplastic junctional or compound nevus
}

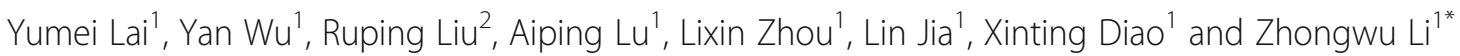

\begin{abstract}
Background/objective: Acral and cutaneous melanomas are usually difficult to accurately diagnose in the early stage, owing to the similarity in clinical manifestations and morphology with those of dysplastic nevus (DN). In this study, we aimed to evaluate the diagnostic value of four-color fluorescence in-situ hybridization (FISH) probes specific to the RREB1,CCND1, and MYB genes, and centromere of chromosome 6, in distinguishing DN and melanoma.

Methods: Fifty one DN and 58 melanoma cases were collected and tested with four-color FISH. Histological features were reviewed and concordant morphologic diagnosis by three pathologists was considered the golden criterion.

Results: Fifty DN and 59 melanoma cases, with 37 melanomas in situ and 22 melanomas in Clark level 2, were confirmed finally; among them, 42 (71.2\%) cases were acral. A comparison of clinicopathological features between the two entities showed that several features were considerably more frequently observed in the melanoma group, including more mitotic figures, stratum corneum pigmentation, lymphocyte infiltration, cell atypia, successive or pagetoid melanocyte growth pattern in the epidermis, larger tumor size, and older age at diagnosis. FISH was positive in 3 (6.0\%) DN and 56 (94.9\%) melanoma cases according to Gerami's criteria. In distinguishing the two groups, the sensitivity of the four-color FISH was $94.9 \%$ and specificity was $94.0 \%$. We found that CCND1 gain was the most sensitive, either in Gerami's or Gaiser's criteria. Further analysis showed that CCND1gain was more obvious in the acral group of melanoma.

Conclusions: We conclude that the four-color FISH test was highly sensitive and specific in distinguishing earlystage acral and cutaneous melanomas from dysplastic nevus in Chinese population, and the most sensitive criterion was the gain of CCND1.
\end{abstract}

Keywords: Acral, Dysplastic nevus, Early stage, Fluorescence in-situ hybridization, Melanoma

\footnotetext{
* Correspondence: zhwuli@hotmail.com

'Department of Pathology, Peking University Cancer Hospital \& Institute, Key

Laboratory of Carcinogenesis and Translational Research (Ministry of

Education/Beijing), Beijing 100142, People's Republic of China

Full list of author information is available at the end of the article
} 


\section{Background}

It is usually challenging for pathologists to diagnose melanoma in the early stage, especially to discriminate melanoma in situ from dysplastic nevus (DN); moreover, it is hard to reach consensus among pathologists, even among the experts. This is because there is no single criterion that can absolutely distinguish melanoma from DN and there are several overlapping clinical and histopathological features of the two entities. In a retrospective study performed by the Dutch Melanoma Working Party in Holland on 1069 melanocytic lesions diagnosed by local doctors, $8 \%$ could not be accurately diagnosed and $14 \%$ cases with initial diagnosis of melanoma were actually benign nevi, while $17 \%$ of cases with initial diagnosis of benign nevi turned out to be malignant later [1]. With respect to some ambiguous melanocytic lesions, neither clinicians nor pathologists can make a clear judgment [2]. On the contrary, patients might sustain physical and mental injuries due to under or over treatment.

Diagnosing melanoma using four-color fluorescence in-situ hybridization (FISH) has been proven to be effective [3-11]. The four-color FISH probes for $6 \mathrm{p} 25$ (RREB1-Ras responsive element-binding protein-1), 6q23 (MYB-myeloblastosis), 11q13 (CCND1,cyclin-D1 or chromosome 11q), and CEP6 (a centromeric reference point on chromosome 6) distinguished melanoma and benign melanocytic lesions according to variation in copy number of the genes, usually gain. Furthermore, the results were highly consistent with histological diagnosis, with the sensitivity of $70.5-100 \%$ and specificity of $90-100 \%$ [3-11]. However, data about its significance in the diagnosis of early-stage melanoma and DN are limited, probably because of limited cases of the two entities and difficulty in diagnosis. Furthermore, data on early lesions in acral has not been reported. In this study, we aimed to evaluate the diagnostic value of four-color fluorescence in-situ hybridization (FISH) probes in distinguishing DN and melanoma.

\section{Materials and methods}

\section{Patients and inclusion criteria}

One hundred and nine surgical excision specimens, 51 DN and 58 early-stage melanoma specimens, either acral or cutaneous, were collected from January 1, 2008 to December 31, 2017 in the Pathology Department of the Peking University Cancer Hospital in Beijing, China. All samples were fixed with neutral buffered formalin, embedded in paraffin, and reviewed by three experienced pathologists. Dysplastic nevus was defined based on the criteria of the Europe Organization for Research and Treatment of Cancer [12]. Specifically, a DN should fulfill at least three of the following four criteria: obvious hyperplasia of nevus cells in the basal layer, irregular nests of nevus cells, nevus cells with a large nucleolus, and nevus cells with lymphocytes or histocytes commonly seen in the background. Furthermore, for all the melanoma cases, Clark level was confined to no more than level 2, that is, only a small part of the papillary layer of dermis was invaded.

\section{Histopathological diagnosis and morphological parameters}

The golden standard to evaluate the sensitivity and specificity of FISH was determined as the histopathological diagnosis by three experienced pathologists blinded to each other and to the results of FISH. For discordant diagnosis, an agreement of two pathologists was considered to be the final diagnosis. Meanwhile, morphologic features including melanocyte growth pattern, cell atypia, maturation, mitotic figures per square millimeter, stratum corneum pigmentation, and lymphocyte infiltration in the interstitial background of the papillary layer were observed under a light microscope, by hematoxylin and eosin $(\mathrm{H} \& \mathrm{E})$ staining. Furthermore, lymphocyte infiltration was further interpreted as mild, moderate, and massive.

\section{FISH detection}

For each case, the most suspicious area of $0.5 \mathrm{~cm}$ in diameter was selected according to $\mathrm{H} \& \mathrm{E}$ staining results for hybridization using the Vysis Melanoma FISH Probe Kit (Abbott Molecular Inc., Abbott Park, IL, USA) specific to RREB1,CCND1, MYB, and centromere of chromosome 6(CEP6). Specifically, FISH was performed with $4-\mu \mathrm{m}-$ thick paraffin sections. The slides were baked at $60{ }^{\circ} \mathrm{C}$ overnight, and then deparaffinized with xylene for $10 \mathrm{~min}$ three times, dexylened with $100 \%$ ethanol for 5 min two times, and washed with water two times. Subsequently, the slides were pretreated with $10 \mathrm{mM}$ citric acid buffer (Pretreatment Solution, Vysis, USA) at $80^{\circ} \mathrm{C}$ for $12 \mathrm{~min}$ after washing with water for three times, followed by pepsin digestion at $37^{\circ} \mathrm{C}$ for $25 \mathrm{~min}$, water wash for two times, and dehydration in an ethanol series for 1 min each. Then, $10 \mu$ Lof probes were added to the tissues and denatured at $75^{\circ} \mathrm{C}$ for $5 \mathrm{~min}$ and hybridized at $37^{\circ} \mathrm{C}$ for $16 \mathrm{~h}$. Thereafter, the slides were washed with 2_SSC/0.3\% NP40 at $71^{\circ} \mathrm{C}$ for $2 \mathrm{~min}$, dehydrated naturally in dark, and counterstained with $10 \mu \mathrm{L}$ of 4,6-diamino-2-phenylindole (DAPI, Vysis, USA). Finally, the slides were stored at $20^{\circ} \mathrm{C}$ after placing over glasses until observation.

FISH was analyzed by a trained physician, who signed the routine cytogenetic reports and was blinded to the results of histological diagnosis. The results were scored according to Gerami's criteria [4]. That is, for each sample, 30 non-overlapping nuclei of tumor cells were counted and considered to be positive if one or more of the following four criteria was satisfied: more RREB1 
(6p25) copies than CEP6 in more than 55\% of cells (gain of RREB1 relative to CEP6), over two copies of RREB1 (6p25) in more than $29 \%$ of cells (gain of RREB1), less $M Y B$ (6q23) copies than CEP6 in more than $40 \%$ of cells (loss of $M Y B$ relative to CEP6), and over two copies of CCND1 (11q13) in more than $38 \%$ of cells (gain of CCND1).

Besides, the criteria of Gerami were compared with those of Gaiser [13], which included another four criteria, and the result was positive if any of the following criteria was fulfilled: an average of more than 2.5 copies of $C C N D 1$ per cell, an average of more than 2.5 copies of $M Y B$ per cell, aberrant copies of RREB1 in more than 63\% of cells, and less MYB (6q23) copies than CEP6 in more than $31 \%$ of cells.

\section{Statistical analysis}

Difference in the age at onset between the two groups of melanoma and nevus and comparison of CCND1 amplification in the mean CCND1 copy number per cell and percentage of cell with more than two copies of CCND1 between acral and cutaneous melanomas were compared using an independent $t$-test. Other differences between the melanoma and nevus groups, or between the acral and cutaneous groups, were analyzed using Pearson's chi-square test. The results with $p$ value of $<.05$ were considered statistically significant. Statistical analyses were performed using SPSS 19.0 for Windows (SPSS Inc., Chicago, IL, USA).

\section{Results}

Clinicopathological features evaluation

After the review by the three pathologists, two cases of melanoma in situ initially diagnosed were found to be dysplastic junctional nevi, while three cases of nevi were revised to be melanoma in situ. Finally, 50 cases of DN and 59 cases of melanoma were confirmed. There were 28 cases of compound nevi and 22 cases of junctional nevi. Among the 59 melanoma cases, 37 were melanoma in situ and 22 were level 2 according to Clark Staging, with Breslow thickness of $0.3-1.0 \mathrm{~mm}$, and without any ulceration. Histological subtypes of the 22 invasive melanomas included 15 acral lentiginous melanomas, 7 superficial spreading melanomas, 1 lentigo maligna melanoma, and 1 spitzoid melanoma.

Clinicopathological features were compared between the melanoma and DN groups and the data are shown in Table 1.The gender ratio of the two entities showed no obvious difference, and so did the tumor site, with the most common sites being acral in both groups. Half of the DNs were more than $6.00 \mathrm{~mm}$ in diameter with the mean diameter of the group being $7.30 \mathrm{~mm}$. However, compared with that in the DN group, the average tumor size in the melanoma group was significantly larger (mean diameter, 11.34 vs. $7.30 \mathrm{~mm}, p=.002$ ), and the age at diagnosis was considerably older (mean age, 47.73 vs. 36.60 years, $p<.001$ ).

Several features were considerably more frequently observed in the melanoma group, including more than one mitotic figures per square millimeter $(39.0 \%$ vs. $2.0 \%$,

Table 1 Clinicopathological feature of dysplastic nevi and early-stage acral and cutaneous melanomas

\begin{tabular}{|c|c|c|c|}
\hline Feature & DN(50 cases), n(\%) & Melanoma(59cases), n(\%) & $p^{\text {a value }}$ \\
\hline Gender ratio (male:female) & $20: 30(0.67: 1)$ & $25: 34(0.74: 1)$ & .802 \\
\hline Age, range (mean) & $3-70(36.60)$ & $9-76(47.73)$ & $<.001^{*}$ \\
\hline \multicolumn{4}{|l|}{ Site } \\
\hline Acral & $31(62.0)$ & $42(71.2)$ & \multirow[t]{4}{*}{.169} \\
\hline Trunk & $9(18.0)$ & $6(10.3)$ & \\
\hline Limb & $9(18.0)$ & $6(10.2)$ & \\
\hline Others & $1(2.0)$ & $5(8.5)$ & \\
\hline mitotic figures $\geq 1 / \mathrm{mm}^{2}$ & $1(2.0)$ & $23(39.0)$ & $<.001^{*}$ \\
\hline Diameter, range (mean, mm) & $1-20(7.30)$ & $2-40(11.34)$ & $.002^{*}$ \\
\hline Stratum corneum pigmentation & $17(34.0)$ & $38(64.4)$ & $.002^{*}$ \\
\hline Moderate to severe lymphocyte infiltration & $6(12.0)$ & $28(47.5)$ & $<.001^{*}$ \\
\hline Moderate to severe cell atypia & $6(12.0)$ & $52(88.1)$ & $<.001^{*}$ \\
\hline \multicolumn{4}{|l|}{ Melanocyte growth pattern in the epidermis } \\
\hline Concessive distribution & $20(40.0)$ & $48(81.4)$ & $<.001^{*}$ \\
\hline Pagetoid spread & $2(4.0)$ & $20(33.9)$ & $<.001^{*}$ \\
\hline Nested distribution & $33(66.0)$ & $44(74.6)$ & .327 \\
\hline Scattered distribution & $37(74.0)$ & $26(44.1)$ & $.002^{*}$ \\
\hline
\end{tabular}

Abbreviations: $D N$ dysplastic nevus, $n$ number

${ }^{a} p$ values from Pearson's chi-square test, independent $t$-test. ${ }^{*} p<.05$, considered statistically significant 
$p<.001)$, stratum corneum pigmentation $(64.4 \%$ vs. $34.0 \%$, $p=.002)$, moderate to severe lymphocyte infiltration ( $47.5 \%$ vs. $12.0 \%, p<.001$ ), moderate to severe cell atypia $(88.1 \%$ vs. $12.0 \%, \mathrm{p}<.001)$, and a successive $(81.4 \%$ vs. $40.0 \%, \mathrm{p}<.001)$ or pagetoid $(33.9 \%$ vs. $4.0 \%, \mathrm{p}<.001)$ melanocyte growth pattern in the epidermis. Maturation of melanocytes was analyzed between specimens of compound nevi and invasive melanomas. The results revealed that all the 22 melanomas were immature, and among the 28 compound nevi specimens, only 3 were immature (100\% vs. $10.71 \%, p<.001)$.

\section{FISH detection}

The results of FISH was positive in 3 (3/50, 6.0\%) DN and 56 (56/59, 94.9\%) melanoma cases (showed in Figs. 1, 2, 3 and 4) according to Gerami's criteria. In distinguishing early-stage melanoma from $\mathrm{DN}$, the sensitivity of FISH was $94.9 \%$, specificity was $94.0 \%$, positive predictive value was $94.9 \%$, and negative predictive value was 94.0\%. Two cases of melanomas primarily misdiagnosed as nevus turned out to be positive and two cases of DNs primarily misdiagnosed as melanoma were negative in FISH detection.
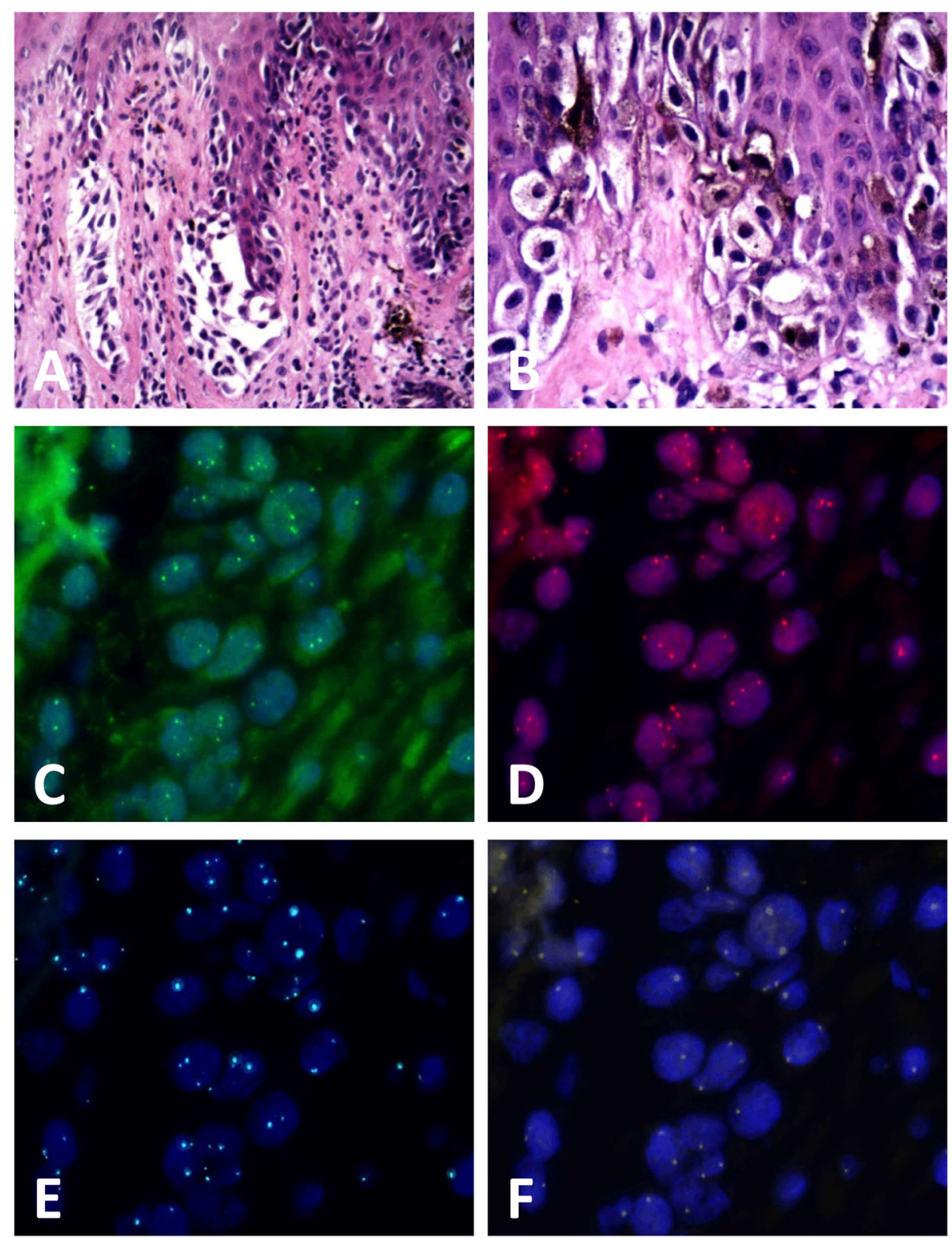

Fig. 1 Case of melanoma in situ positive by fluorescence in-situ hybridization (FISH) detection. a Melanocytes proliferated successively or in nests in the basal layer of the epidermis (hematoxylin and eosin, H\&E staining), (b) high-power magnification showed moderate to severe atypia of the melanocytes(H\&E staining), (c-f) FISH images showed the (c) gain of CCND1 (green signals), (d) gain of RREB1 (red signals), and (f) loss of MYB (gold signals) relative to CEP6 (aqua signals). While the gain of RREB1(d) relative to CEP6 (e) was not observed 

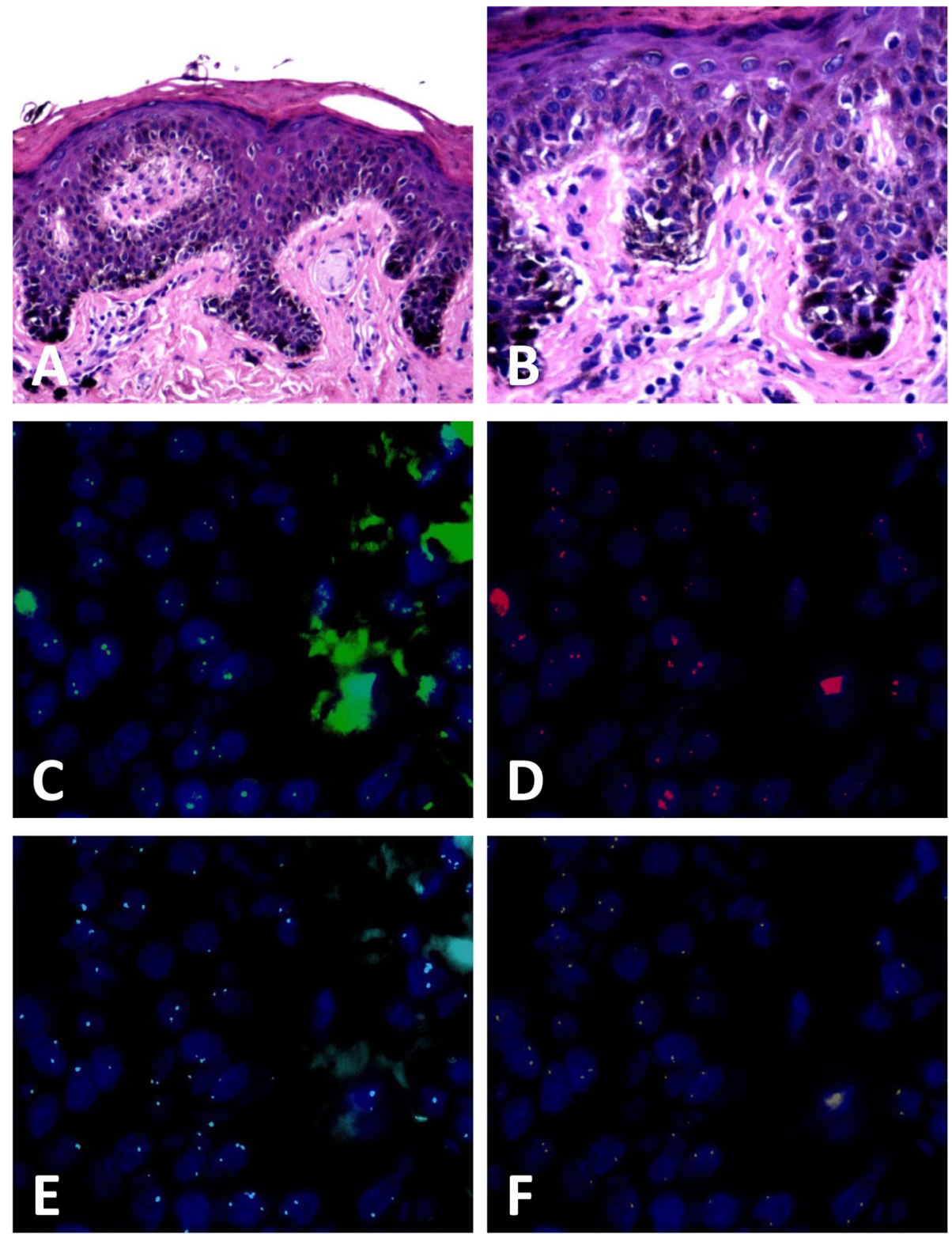

Fig. 2 Case of dysplastic junctional nevus negative by fluorescenc e in-situ hybridization (FISH) detection. a Melanocytes proliferated successively or scattered in the basal layer of the epidermis (hematoxylin and eosin, H\&E staining), (b)high-power magnification showed mild atypia of the melanocytes(H\&E staining), (c-f) FISH images showed normal copies of CCND1 (c, green signals), RREB1 (d, red signals), CEP6 (e, aqua signals), and MYB (f, gold signals)

Among the 56 melanoma cases positive by FISH detection, only one case fulfilled all four criteria of Gerami, and nearly half of them fulfilled three criteria, specifically, 18 cases met the criteria "gain of CCND1", "gain of $R R E B 1$ ", and "loss of $M Y B$ relative to CEP6", and 8 cases met "gain of RREB1", "gain of CCND1", and "gain of RREB1 relative to CEP6". Most of the remaining cases satisfied two of the criteria, with 15 of them satisfying "gain of CCND1" and "gain of RREB1", 4 of them meeting "gain of CCND1" and "loss of $M Y B$ relative to
CEP6", and 1meeting both "gain of RREB1" and "loss of $M Y B$ relative to CEP6". While 9 cases fulfilled only one criterion, that is, 8 cases were positive for "gain of CCND1", and one positive for "gain of RREB1". Of the $3 \mathrm{DN}$ positive cases by FISH assay, all fulfilled both "gain of RREB1" and "gain of CCND1", and one of them fulfilled the criterion of "loss of $M Y B$ relative to CEP6" as well.

We also analyzed the number and percentage of cases meeting each criterion of Gerami and Gaiser (Table 2). 

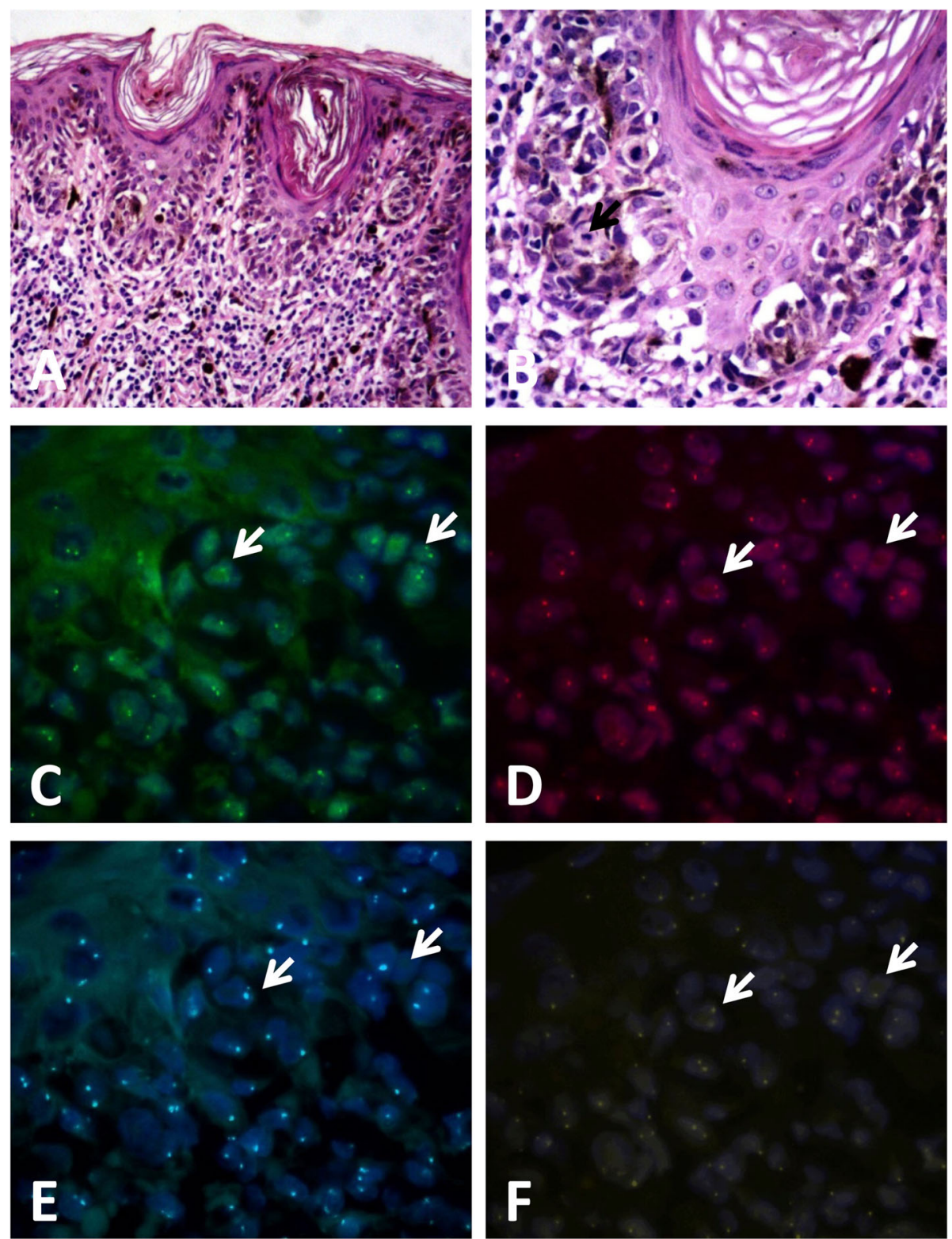

Fig. 3 Case of melanoma in situ negative by fluorescence in-situ hybridization (FISH) detection. a Melanocytes proliferated successively or in nests in the basal layer of the epidermis (hematoxylin and eosin, H\&E staining),(b) high-power magnification showed moderate atypia of the melanocytes with mitotic figures, which were easy to identify (black arrow)(H\&E staining), (c-f) FISH images showed normal copies ofCCND1 (c, green signals), RREB1 (d, red signals), CEP6 (e, aqua signals), and MYB (f, gold signals) in tumor cells (white arrows)

The results showed that the criterion associated with the gain of CCND1was the most frequent to fulfill in the two criterion systems, with the positive rate of 91.5 and $88.1 \%$, respectively, in the melanoma group and both $6.0 \%$ in the DN group, followed by Gerami criterion "gain of RREB1" with the positive rate of $74.6 \%$ in the melanoma group.

The sensitivity and specificity of each criterion of Gerami and Gaiser were studied (Table 3). According to Gerami's criteria, the most sensitive criterion was "gain of CCND1" (91.5\%), followed by "gain of RREB1"(74.6\%), and the most specific criterion was "gain of RREB1 relative to CEP6" (100\%). If FISH was interpreted with Gaiser's criteria, the overall sensitivity was $93.2 \%$ and specificity was $90.0 \%$, and the most sensitive criterion was the gain of CCND1, which was $88.1 \%$; the sensitivity of the other three criteria was very close.

We compared the differences in CCND1 amplification between the acral and cutaneous groups of melanoma 

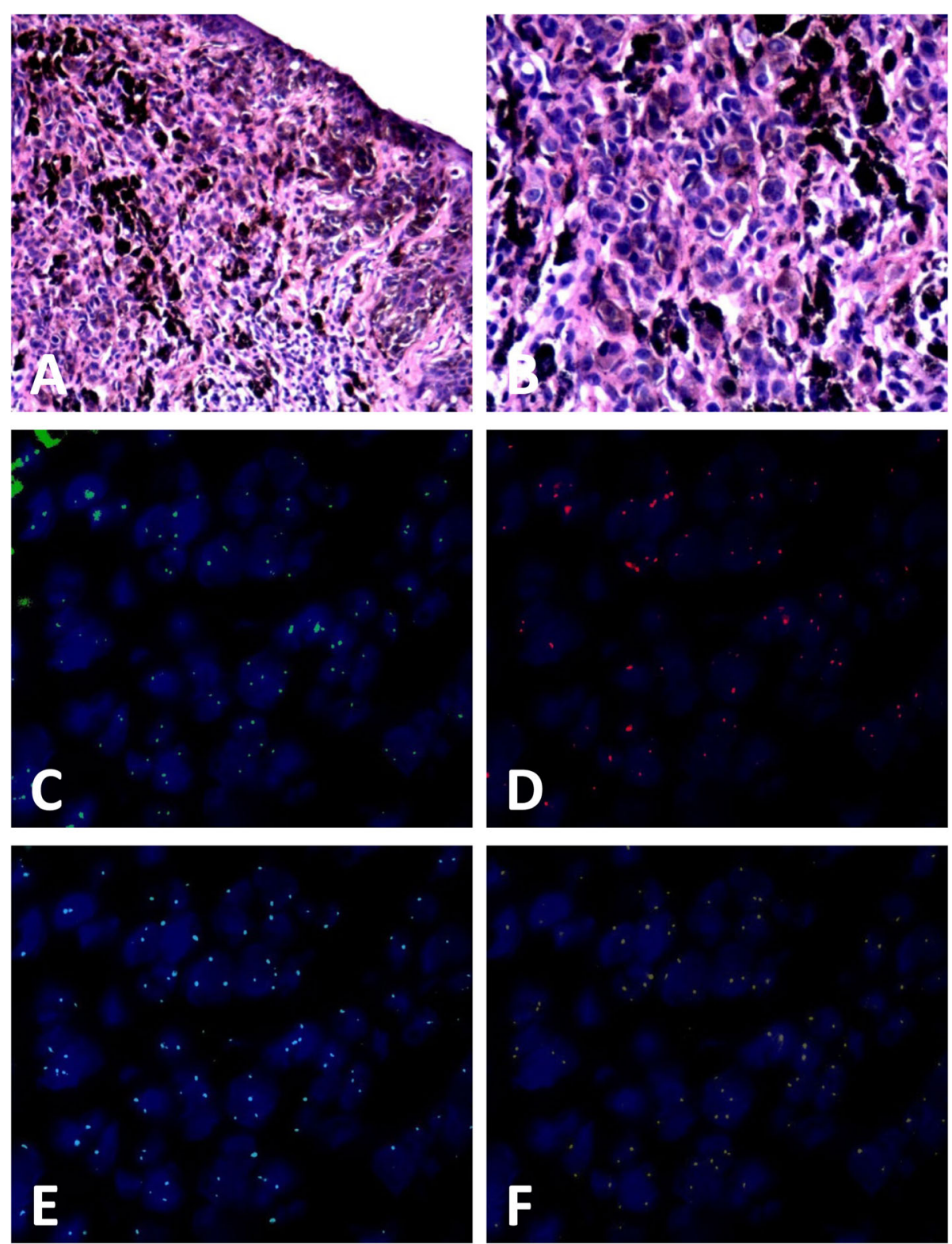

Fig. 4 Case of compound nevus positive by fluorescence in-situ hybridization (FISH) detection. a Melanocytes scattered in the basal layer of the epidermis and proliferated without maturation in the dermis (hematoxylin and eosin, H\&E staining), (b) high-power magnification showed mild atypia of the melanocytes and no mitotic Fig. (H\&E staining),(c-f) FISH images showed the (c) gain of CCND1 (green signals), (d) gain of RREB1 (red signals), while the gain of RREB1(d, red signals) relative to CEP6 (e, aqua signals) or loss of MYB (f, gold signals) relative to CEP6 (e, aqua signals) was not observed

(Table 4) and showed that CCND1was more amplified in the acral group. Specifically, the mean CCND1 copy number per cell and percentage of cells with CCND1 amplification were considerably higher in the acral melanoma group, with the average of 3.74-3.03 ( $p=.073)$ and 76.74$58.67 \%(p=.007)$, respectively. Cases meeting the criteria associated with CCND1amplification were also more in the acral group, although the differences were not so obvious.

\section{Discussion}

The results of the present study showed that the four-color FISH detection was highly sensitive and specific in differentiating early-stage melanoma from DN in Chinese population, and the gain of CCND1presented the highest sensitivity. To the best of our knowledge, this is the first study to analyze the usage of FISH focusing on early-stage melanomas 
Table 2 Number and percentage of cases meeting each diagnostic criterion of Gerami and Gaiser for dysplastic nevi and early-stage acral and cutaneous melanomas

\begin{tabular}{|c|c|c|}
\hline & $\mathrm{DN}, \mathrm{n}(\%)$ & melanoma, n(\%) \\
\hline Total, n & 50 & 59 \\
\hline \multicolumn{3}{|l|}{ Gerami's criteria } \\
\hline RREB1 > CEP6 (> 55\%) & 0 & $9(15.3)$ \\
\hline RREB1 > 2 (> 29\%) & $3(6.0)$ & $44(74.6)$ \\
\hline MYB<CEP6 (> 40\%) & $1(2.0)$ & $24(40.7)$ \\
\hline CCND1 > 2 (> 38\%) & $3(6.0)$ & $54(91.5)$ \\
\hline FISH positive & $3(6.0)$ & $56(94.9)$ \\
\hline \multicolumn{3}{|l|}{ Gaiser's criteria } \\
\hline RREB1 aberrant (> 63\%) & $3(6.0)$ & $28(47.5)$ \\
\hline MYB<CEP6 (> 31\%) & $1(2.0)$ & $30(50.8)$ \\
\hline MYB $\geq 2.5$ & $3(6.0)$ & $28(47.5)$ \\
\hline CCND1 $\geq 2.5$ & $3(6.0)$ & $52(88.1)$ \\
\hline FISH positive & $5(10.0)$ & 55 (93.2) \\
\hline
\end{tabular}

Abbreviations: $D N$ dysplastic nevus, $n$ number

in Chinese patients, and the majority of which were acral melanomas in situ.

The incidence of melanoma in East Asia and Southeast Asia is significantly lower than that in North America and Europe. According to the GLOBOCAN 2012 statistics, the incidence of age-standardized melanomas is $0.4-0.5 / 100000$ persons in East and Southeast Asia, while 8.6-13.8/100000 persons in Europe and North America [14]. However, due to the huge population, the burden of melanoma should not be

Table 3 Sensitivity and specificity of each criterion of Gerami and Gaiser in differentiating dysplastic nevi and early-stage acral and cutaneous melanomas

\begin{tabular}{lll}
\hline & Sensitivity & Specificity \\
\hline Gerami's criteria & & \\
RREB1 > CEP6 (> 55\%) & $15.2 \%$ & $100 \%$ \\
RREB1 > 2 (> 29\%) & $74.6 \%$ & $94.0 \%$ \\
MYB <CEP6 (> 40\%) & $40.7 \%$ & $98.0 \%$ \\
CCND1 > 2 (> 38\%) & $91.5 \%$ & $94.0 \%$ \\
Total $^{a}$ & $94.9 \%$ & $94.0 \%$ \\
Gaiser's criteria & & \\
RREB1 aberrant (> 63\%) & $47.5 \%$ & $94.0 \%$ \\
MYB<CEP6 (> 31\%) & $50.8 \%$ & $98.0 \%$ \\
MYB $\geq 2.5$ & $47.5 \%$ & $94.0 \%$ \\
CCND1 22.5 & $88.1 \%$ & $94.0 \%$ \\
Total $^{a}$ & $93.2 \%$ & $90.0 \%$ \\
\hline
\end{tabular}

a total sensitivity and specificity of the four criteria, fulfilling any of the four criteria is considered positive ignored in Asia. Moreover, in Asia, especially in China, melanoma is often neglected and diagnosed late, and therefore, patients with melanoma in these areas are usually in an advanced stage with poor prognosis $[15,16]$. Therefore, an early diagnosis of melanoma is very important.

Histologically, early-stage melanoma and DN can overlap, especially in the acral area. For example, melanocytes in acral DN are usually solitary and show mild to moderate cell atypia and lymphocyte or histocyte infiltration [17]. On the contrary, early-stage acral melanoma mostly manifests as solitary atypical melanocytes in the dermo-epidermal junction area [18]. In this study, we found that moderate to severe cell atypia, successive or pagetoid melanocyte growth pattern in the epidermis, more than one mitotic figures per square millimeter, moderate to severe lymphocyte infiltration, and stratum corneum pigmentation were significantly more often observed in early-stage acral and cutaneous melanomas than in nevi of such areas. However, distinguishing DNs from early melanomas in areas of acral is still difficult. Most of the above features in favor of differential diagnosis were observed in only less than half of the melanoma group patients in our study. Furthermore, none of the features was specific enough to diagnose a melanoma.

Four-color FISH probe is a useful tool to assist the diagnosis of melanoma. The results of various studies are not consistent, with a sensitivity of $70.5-100 \%$ and specificity of $90-100 \%$ [3-11]. The specificity in our study was similar to that reported previously, but the sensitivity was considerably higher [8-11]. The differences in sensitivity might be associated with the site and ethnic differences, and tumor stage. Studies have shown significant differences in genotypes between acral and cutaneous melanomas, also among different ethnic populations [19-22].

We found that the CCND1gene gain was more common in the acral group than in the cutaneous group. This result was in accordance with those of studies based on comparative genomic hybridization and immunohistochemistry $[22,23]$. However, the most sensitive criterion in our study was the gain of CCND1, which was considerably higher than that in previous studies of acral melanomas, including a study based on the same ethnic population, in which the majority of cases was advanced melanomas and the most sensitive criterion was the gain of RREB1 instead [10, 24]. Interestingly, in Su's study [10], of the seven cases of acral melanoma in situ, the most sensitive criterion was also the gain of $C C N D 1$, specifically, 5 cases were positive for $C C N D 1$; of the two negative cases, one showed $33 \%$ of cells gaining CCND1 gene, close to the positive threshold of $38 \%$, 
Table 4 Comparison of the number and percentage of cases meeting each criterion of Gerami and Gaiser, and CCND1 amplification between the acral and non-acral groups of melanoma

\begin{tabular}{|c|c|c|c|}
\hline & Acral group, $\mathrm{n}(\%)$ & Non-acral group, $\mathrm{n}(\%)$ & $p^{\mathrm{a}}$ value \\
\hline Total & 42 & 17 & \\
\hline \multicolumn{4}{|l|}{ Gerami's criteria } \\
\hline RREB1 > CEP6 (> 55\%) & $6(14.3)$ & $3(17.6)$ & 1.000 \\
\hline RREB1 > 2 (> 29\%) & $33(78.6)$ & $11(64.7)$ & .437 \\
\hline MYB<CEP6 (> 40\%) & $19(45.2)$ & $5(29.4)$ & .262 \\
\hline CCND1 > 2 (> 38\%) & $40(95.2)$ & $14(82.4)$ & .274 \\
\hline FISH positive & $41(97.6)$ & $15(88.2)$ & .197 \\
\hline \multicolumn{4}{|l|}{ Gaiser's criteria } \\
\hline RREB1 aberrant (>63\%) & $22(52.4)$ & $6(35.3)$ & .234 \\
\hline MYB<CEP6 (> 31\%) & $21(50.0)$ & $9(52.9)$ & .838 \\
\hline$M Y B \geq 2.5$ & $21(50.0)$ & $7(41.2)$ & .539 \\
\hline CCND1 $\geq 2.5$ & $39(92.9)$ & $13(76.5)$ & .187 \\
\hline FISH positive & $40(95.2)$ & $15(88.2)$ & .691 \\
\hline CCND1 copy numbers per cell,mean & 3.74 & 3.03 & .073 \\
\hline Percentage of cells with CCND1 > 2, mean & $76.74 \%$ & $58.67 \%$ & $.007^{*}$ \\
\hline
\end{tabular}

Abbreviations: $n$ number

${ }^{a} p$ values from Pearson's chi-square test, independent $t$-test. ${ }^{*} p<.05$, considered statistically significant

while the other one was negative in the FISH assays with all the probes, including the $M Y C$ and $C D K N 2 A$ probes. Therefore, we inferred that the CCND1gene gain is one of the most frequent genetic changes in early-stage acral melanomas in the Chinese population, and it was helpful in diagnosing acral melanoma in situ. Studies have indicated frequent amplification of genes involved in the CDK4 pathway including CCND1 in acral melanoma [21, 25], indicating the potential for CDK4/6 inhibitors. However, the significance of CCND1 gain in the occurrence or in situ state of acral melanoma needs further studies.

Considering that false positivity and negativity still exited in a subset of cases, FISH detection should be supplemented with histopathological evaluation and should not replace conventional microscopy in discriminating melanomas from DNs. Three nevus cases were positive for the FISH assay in our study. Further analysis showed that all of the four genes (CCND1, RREB1,MYB and CEP6) manifested three copies, suspicious of polyploidy, which is the most common false positivity in spitz nevi. None of the three cases was spitz nevus in morphology, though. However, as it is possible that genetic changes might precede morphological changes, we recommend that for those DN cases with positive FISH results, complete resection with enough negative margin and close follow-up might be the appropriate treatment. Three melanoma cases with negative FISH results were found in this study. Since we only tested four genes, it is comprehensible to understand that these cases might be associated with other gene alterations such as $M Y B$ loss and $C D K N 2 A$ homozygous deletion. A combination with $M Y B$ or $C D K N 2 A$ probes may increase the sensitivity [10].

A limitation of the study is that we lacked prognosis information and regarded the histopathological diagnosis as the golden standard. Considering the good prognosis of early-stage melanoma, long time follow-up for decades is needed to elucidate its biological behavior, and maybe then we can investigate and distinguish the two groups more thoroughly. Another limitation is that we have not confirmed the gene alterations of the three false-negative melanoma cases with a MYC or CDKN2A probes or a 5 or 7 color FISH probes, since the former probes are unavailable for us.

\section{Conclusions}

To conclude, with high sensitivity and specificity, the four-color FISH technique was a valuable ancillary tool to distinguish early-stage acral and cutaneous melanomas from DNs in Chinese population. Furthermore, the CCND1 gene gain was the most sensitive criterion of melanomas in this cohort. A combination of the fourcolor FISH results with histological analysis was necessary to explain the positive or negative significance of FISH. For DN cases with positive FISH results, complete 
resection with sufficient negative margin and close follow-up might be the appropriate treatment.

\section{Abbreviations}

DN: Dysplastic nevus; FISH: Fluorescence in-situ hybridization; H\&E: Hematoxylin and eosin

\section{Acknowledgments}

We thank Dr. Jianying Liu and Dr. Jing Su for providing suggestions regarding the FISH technique on melanoma.

\section{Authors' contributions}

Zhongwu Li designed the study, reviewed tumor morphology, and revised the manuscript; Yumei Lai interpreted the FISH results, performed statistical analyses, and wrote the manuscript; Yan Wu and Ruping Liu collected the data and carried out data analysis; Yan Wu and Aiping Lu reviewed the morphology; Lixin Zhou directed the FISH test; and Lin Jia and Xinting Diao performed FISH detection. All authors read and approved the final manuscript.

\section{Funding}

This work was supported by the Capital's Funds for Health Improvement and Research [grant number 2018-2-1022]; the Beijing Municipal Science and Technology Commission NOVA Program [grant number 2010 B033]; the Beijing Municipal Science and Technology Commission Capital Characteristic Clinical Application Research [grant number Z141107002514077]; the National Natural Science Foundation of China [grant number 61501039]; and the Beijing Cancer Hospital [grant number 13-11].

\section{Availability of data and materials}

Not applicable.

\section{Ethics approval and consent to participate}

Written informed consent from all the patients and approval from the Ethics Committee of the Peking University Cancer Hospital were obtained.

\section{Consent for publication}

Not applicable.

\section{Competing interests}

The authors declare that they have no competing interests.

\section{Author details}

'Department of Pathology, Peking University Cancer Hospital \& Institute, Key Laboratory of Carcinogenesis and Translational Research (Ministry of Education/Beijing), Beijing 100142, People's Republic of China. 'Beijing Institute of Graphic Communication, Beijing 102600, People's Republic of China.

\section{Received: 24 October 2019 Accepted: 6 February 2020}

\section{Published online: 11 May 2020}

\section{References}

1. Veenhuizen KC, De Wit PE, Mooi WJ, Scheffer E, Verbeek AL, Ruiter DJ. Quality assessment by expert opinion in melanoma pathology: experience of the pathology panel of the Dutch melanoma working party. J Pathol. 1997:182:266-72.

2. Lodha S, Saggar S, Celebi JT, Silvers DN. Discordance in the histopathologic diagnosis of difficult melanocytic neoplasms in the clinical setting. J Cutan Pathol. 2008:35:349-52.

3. Clemente C, Bettio D, Venci A, Scopsi L, Rao S, Ferrari A, et al. A fluorescence in situ hybridization (FISH) procedure to assist in differentiating benign from malignant melanocytic lesions. Pathologica. 2009;101:169-74.

4. Gerami P, Jewell SS, Morrison LE, Blondin B, Schulz J, Ruffalo T, et al. Fluorescence in situ hybridization (FISH) as an ancillary diagnostic tool in the diagnosis of melanoma. Am J Surg Pathol. 2009;33:1146-56

5. Morey AL, Murali R, McCarthy SW, Mann GJ, Scolyer RA. Diagnosis of cutaneous melanocytic tumours by four-colour fluorescence in situ hybridisation. Pathology. 2009;41:383-7.

6. Abasolo A, Vargas MT, Rios-Martin JJ, Trigo I, Arjona A, Gonzalez-Campora R Application of fluorescence in situ hybridization as a diagnostic tool in melanocytic lesions, using paraffin wax-embedded tissues and imprintcytology specimens. Clin Exp Dermatol. 2012;37:838-43.

7. Dalton SR, Gerami P, Kolaitis NA, Charzan S, Werling R, LeBoit PE, et al. Use of fluorescence in situ hybridization (FISH) to distinguish intranodal nevus from metastatic melanoma. Am J Surg Pathol. 2010;34:231-7.

8. Fang Y, Dusza S, Jhanwar S, Busam KJ. Fluorescence in situ hybridization (FISH) analysis of melanocytic nevi and melanomas: sensitivity, specificity, and lack of association with sentinel node status. Int J Surg Pathol. 2012;20: 434-40.

9. Gerami P, Li G, Pouryazdanparast P, Blondin B, Beilfuss B, Slenk C, et al. A highly specific and discriminatory FISH assay for distinguishing between benign and malignant melanocytic neoplasms. Am J Surg Pathol. 2012;36: 808-17.

10. Su J, Yu W, Liu J, Zheng J, Huang S, Wang Y, et al. Fluorescence in situ hybridisation as an ancillary tool in the diagnosis of acral melanoma: a review of 44 cases. Pathology. 2017:49:740-9.

11. Vergier B, Prochazkova-Carlotti M, de la Fouchardiere A, Cerroni L, Massi D, De Giorgi $V$, et al. Fluorescence in situ hybridization, a diagnostic aid in ambiguous melanocytic tumors: European study of 113 cases. Mod Pathol. 2011;24:613-23.

12. de Wit PE, van't Hof-Grootenboer B, Ruiter DJ, Bondi R, Brocker EB, Cesarini JP, et al. Validity of the histopathological criteria used for diagnosing dysplastic naevi. An interobserver study by the pathology subgroup of the EORTC malignant melanoma cooperative group. Eur J Cancer. 1993:29:8319.

13. Gaiser T, Kutzner H, Palmedo G, Siegelin MD, Wiesner T, Bruckner T, et al. Classifying ambiguous melanocytic lesions with FISH and correlation with clinical long-term follow up. Mod Pathol. 2010;23:413-9.

14. Ferlay J, Soerjomataram I, Dikshit R, Eser S, Mathers C, Rebelo M, et al. Cancer incidence and mortality worldwide: sources, methods and major patterns in GLOBOCAN 2012. Int J Cancer. 2015;136:E359-86.

15. He J, Chen W. Annual report of China Cancer registry, 2015. Beijing: Tsinghua University Press; 2017.

16. He J, Chen W. Annual report of China Cancer Registry, 2016. Beijing: People's Medical Publishing House Press; 2017.

17. Fallowfield ME, Collina G, Cook MG. Melanocytic lesions of the palm and sole. Histopathology. 1994;24:463-7.

18. Bravo Puccio F, Chian C. Acral junctional nevus versus acral lentiginous melanoma in situ: a differential diagnosis that should be based on clinicopathologic correlation. Arch Pathol Lab Med. 2011;135:847-52.

19. Chi Z, Li S, Sheng X, Si L, Cui C, Han M, et al. Clinical presentation, histology, and prognoses of malignant melanoma in ethnic Chinese: a study of 522 consecutive cases. BMC Cancer. 2011;11:85.

20. Guo J, Qin S, Liang J, Lin T, Si L, Chen X, et al. Chinese guidelines on the diagnosis and treatment of melanoma (2015 edition). Ann Transl Med. 2015; 3:322.

21. Yeh I, Jorgenson E, Shen L, Xu M, North JP, Shain AH, et al. Targeted genomic profiling of acral melanoma. J Natl Cancer Inst. 2019;111(10):106877.

22. Bastian BC, Kashani-Sabet M, Hamm H, Godfrey T, Moore DH 2nd, Brocker $E B$, et al. Gene amplifications characterize acral melanoma and permit the detection of occult tumor cells in the surrounding skin. Cancer Res. 2000;60: 1968-73.

23. Ibrahim ZA, Narihan MZ, Ojep DN, Soosay AE, Pan KL. Cyclin D1 expression in acral melanoma: a case control study in Sarawak. Malays J Pathol. 2012; 34:89-95.

24. Diaz A, Puig-Butille JA, Valera A, Munoz C, Costa D, Garcia-Herrera A, et al. TERT and AURKA gene copy number gains enhance the detection of acral lentiginous melanomas by fluorescence in situ hybridization. J Mol Diagn. 2014;16(2):198-206.

25. Kong Y, Sheng X, Wu X, Yan J, Ma M, Yu J, et al. Frequent genetic aberrations in the CDK4 pathway in acral melanoma indicate the potential for CDK4/6 inhibitors in targeted therapy. Clin Cancer Res. 2017;23:6946-57.

\section{Publisher's Note}

Springer Nature remains neutral with regard to jurisdictional claims in published maps and institutional affiliations. 